Conclusion: Theatre staff report an initial challenging learning curve with EES. With time however the theatre team satisfaction levels are higher due to greater awareness and involvement with the surgical procedure.

Learning points: The theatre team shows high levels of satisfaction with EES.

doi:10.1017/S0022215116005090

\section{ID: IP013}

Sonotubometry using perfect sequences: clinical results of the $\mathbf{1 0 5}$ healthy subjects

Presenting Author: Vilma Beleskiene

Vilma Beleskiene

Vilnius University Hospital Santariskiu Clinics

\section{Learning Objectives:}

Objectives: The aim of this study was to establish the rate variation of sonotubometric measurements using a specific broadband class of signals, the so-called perfect sequences (PSEQ) among healthy adults and to identify an optimal and technically simple test to provoke Eustachian tube (ET) openings.

Methods: Sonotubometry was performed on 105 healthy adult subjects. Three different consecutive maneuvers were performed for ET opening: dry swallowing, water swallowing (a small $(2 \mathrm{ml})$ and a large $(5 \mathrm{ml})$ water bolus). Values of the amplitude and duration of each measured ET opening were calculated.

Results: 6,300 measurements were performed. Sonotubometric ET openings were detected for all subjects but not for each measurement. 6,180/6,300 measurements (98.1\%) objective ET openings were registered. Mean ET opening duration time and the mean sound amplitude were similar for all performed test and are $270(\mathrm{SD} \pm 96) \mathrm{ms}$, $13.48(\mathrm{SD} \pm 6.57) \mathrm{dB}$.

Conclusion: sonotubometry based on PSEQ stimuli is a reliable methodology to assess the Eustachian Tube opening function in healthy subjects. Mean ET opening duration time and the mean sound wave amplitude were similar performing all analysed tests, hence might be concluded, that dry (saliva) and water swallowing are a reliable sonotubometric maneuvers and may be used examining ET opening function. Size of a sip during water swallowing does not affect the sonotubometry result. All maneuvers can be equally used as the optimal maneuver, and we think that water swallow is most comfortable for the subject.

\section{doi:10.1017/S0022215116005107}

\section{ID: IP014}

Devices for restoring hearing in the sequelae of cholesteatoma
Presenting Author: Millo Achille Beltrame

Millo Achille Beltrame

Centro Clinico Multispecialistico

Rovereto

Learning Objectives: Possible solution to restore the hearing using bone conduction implants and middle ear implants solutions. The presentation illustrates different situations, in patients where the hearing loss changed post cholesteatoma's surgery.

The hearing loss in patients with history of Cholesteatoma,have multifarious features that depend either by the damaging disease activity or by the result of the surgery. The majority of them suffer from conductive hearing losses because the ossicles have been destroyed by the disease or surgically removed. The hearing has to be restored, reconstructing the ossicular chai$\mathrm{n}$ (ossiculoplasty)or, in other cases, with hearingimplants. Frequently patients with previous cholesteatoma, show a mixed hearing loss, due to the aging or toxicity of the disease. A limited number presents a profound deafness, or anacusis due to a cholesteatoma's invasion into the labyrinth or by iatrogenic damage during the surgery. All these outcomes can be corrected with auditory implants inserted either in the middle ear or in the cochlea. Subjects that have CWU and CWD tympanoplasty done and good bone hearing threshold were treated with BONEBRIDGE device in the retro-sigmoid site, in order to avoid any future contamination in case of a disease recurrence or infections in the middle ear. In mixed hearing losses, a Vibrabt Soundbridge system has been preferred to place the FMT over the stapes, if present, or onto the footplate or on the round window. In CWD cases the round window membrane has been always the site of choice. When the cavity is completely clean and dry a VSB could be implanted with the FMT on the $\mathrm{RW}$ pays attention of the positioning of the conductor link, far from the diseased area and in a channel covered with bone pâté. Doing a cholesteatoma removal and a middle ear implantation on the same surgical session is not always the best option. There are situations where the procedure requires two steps: cholesteatoma removal first and implantation later. Always an open external ear canal is mandatory in order to check with otoscopy the condition of the middle ear cavity in the next visits.Finally,in patients with profound hearing losses, a cochlear implant was implanted via retro-sub-facial approach.

doi:10.1017/S0022215116005119

\section{ID: IP015}

The Chorda Tympani Nerve Degenerates during Chronic Otitis Media. An Electrone Microscopy Study

\section{Presenting Author: Katarina Berling Holm}

Katarina Berling Holm ${ }^{1}$, Magnus von Unge ${ }^{2}$, Paula Mannström ${ }^{3}$, Mats Ulfendahl ${ }^{3}$, Niklas Danckwardt Lillieström ${ }^{4}$

${ }^{1}$ Center for Clinical Research, Landstinget Västmanland, Västerås, Sweden, ${ }^{2}$ Dept. of Otorhinolaryngology, Akershus University Hospital and University of Oslo, Norway, ${ }^{3}$ Dept. of Neuroscience, Karolinska Institutet, 
Stockholm, Sweden, ${ }^{4}$ ENT department Uppsala Academic Hospital, Uppsala University

Learning Objectives: Patients with chronic inflammatory middle ear diseases can experience taste disturbance before surgery due to the degenerative capacity of the inflammatory process.

Background: The important nerve of taste, the chorda tympani nerve, runs uncovered through the middle ear. This location predisposes it to become affected by bacterial toxins, enzymes and mechanical damage in various forms of middle ear pathology, such as chronic otitis media and cholesteatoma. A difference between inflammatory diseases, such as chronic suppurative otitis media and cholesteatoma, and noninflammatory diseases, such as otosclerosis, regarding taste disturbance preoperatively and symptoms postoperatively have been noticed. The present study aims to investigate ultrastructural changes of chorda tympani in inflammatory middle ear disease as compared with normal.

Methods: Five chorda tympani specimens were collected from healthy middle ears of patients subjected to surgery for acoustic neuroma to be used as normal controls, and five from middle ears with chronic otitis media or cholesteatoma where the nerve could not be saved during the operation. Light microscopy and electron microscopy were used to identify signs of pathological processes.

Results: Ultrastructural changes that implicate inflammatory changes and degeneration were found in all five nerves from ears with chronic otitis media and cholesteatoma. There were signs of proliferation of connective tissue of the endoneurium, disorganization and demyelination of axons, vacuolar degeneration of the axons, myelin sheath disintegration and edema. As a sign of regeneration capacity there was occurrence of sprouting in CTN from ears with inflammatory diseases.

Conclusion: Chorda tympani nerves from ears with chronic inflammatory middle ear disease exhibit structural signs of deterioration that correlates well to taste disturbances.

There were signs of nerve regeneration that could explain the ability of taste recovery.

doi:10.1017/S0022215116005120

\section{ID: IP016}

Use of Bioactive glass S53P4 in mastoid

and epitympanic obliteration: our

experience in 74 cases

Presenting Author: Daniele Bernardeschi

Daniele Bernardeschi, Olivier Sterkers

Pitié-Salpêtrière Hospital

Learning Objectives:

Objective: to present our experience with the use of Bioactive glass S53P4 in ear surgery
Material and Methods: Seventy-four cases (72 adult patients, 2 operated bilaterally) operated from May 2013 to December 2015 in a tertiary referral center. All but 10 cases were revision surgeries (mean previous operation $=$ 2 ). The mean pre-operative hearing threshold was $57 \pm 18$ and $29 \pm 15 \mathrm{~dB}$ for air conduction and bone conduction respectively. All patients underwent mastoid and epitympanic obliteration in a single stage CWD $(n=60)$ or CWU $(\mathrm{n}=14)$ tympanomastoidectomy. Intraoperative bacteriological test was performed for all patients. Anatomical and functional results were evaluated 3 months and 1 year after surgery, and a CT scan \pm MRI was performed 1 year after surgery. Quality of life measured with the GBI and specific questionnaire was performed 1 year after surgery.

Results: Cholesteatoma was found in 48 cases; Bacteriological tests showed some bacteria and/or fungus in 43 cases. At 3 months all but 2 patients had a wellhealed EAC and intact tympanic drum. Two cases of uncovered granules in the EAC underwent revision surgery for recovering of the granules with cartilage. At 1 year $(n=$ 41 ), anatomical results remained stable with no cases of recurrent cholesteatoma. Regarding the hearing, one year after surgery $(n=41)$ the mean hearing threshold was $46 \pm 22$ and $27 \pm 17 \mathrm{~dB}$ for air conduction and bone conduction respectively. CT scan $(n=41)$ and MRI $(n=17)$ showed no residual cholesteatoma inside or near the obliteration. GBI and specific questionnaire $(\mathrm{n}=41)$ showed an improvement in the QOL (mean total score $=28$ ).

Conclusion: The bioactive glass S53P4 is a well-tolerated biomaterial for primary or revision chronic otitis surgery, as shown by the absence of revision surgery for removal of the granules even in case of surgery in infected ears. Hearing results depend mainly on the number of previous surgeries, and the patient's quality of life is improved after operation.

doi:10.1017/S0022215116005132

\section{ID: IP017}

A systematic review of the epidemiological

relationship of mucosal otitis media, tympanic retraction, and cholesteatoma

Presenting Author: Mahmood Bhutta Mahmood Bhutta

Royal National Throat Nose and Ear Hospital

Learning Objectives: To better understand the relationship of squamous forms of otitis media to mucosal disease, based upon a systematic review of longitudinal epidemiological studies. To use these data to create a map of disease relationships.

Introduction: Clinical experience suggests that cholesteatoma often arises in individuals with a history of prior mucosal otitis media, or a history of tympanic retraction. I set out to exploit existing longitudinal studies to ascertain the relation of these disease entities, specifically to assess the relation of mucosal disease to tympanic retraction and cholesteatoma. 